\title{
Fault Detection and Classification for Slider Attachment Process using Convolution Neural Network
}

\author{
Thanaporn Thamcharoen \\ School of Mechanical Engineering, \\ Institute of Engineering, Suranaree \\ University of Technology, \\ Nakhon Ratchasima 30000, Thailand \\ email: thanaporn.cme@gmail.com
}

Jiraphon Srisertpol

School of Mechanical Engineering,

Institute of Engineering, Suranaree University of Technology,

Nakhon Ratchasima 30000, Thailand email:jiraphon@sut.ac.th

\author{
Prathan Chommuangpuck \\ Western Digital Thailand Co. Ltd. \\ Bang Pa-in Industrial Estate, Bang Pa- \\ In, Phra Nakhon Si Ayutthaya 13160, \\ Thailand \\ email:pranthan8137@ hotmail.com
}

\author{
Jakawat Deeying \\ Western Digital Thailand Co. Ltd. \\ Bang Pa-in Industrial Estate, Bang Pa- \\ In, Phra Nakhon Si Ayutthaya 13160, \\ Thailand \\ email: jakawat.deeying@gmail.com
}

\begin{abstract}
Hard Disk Drive (HDD) utilizes automation machines for the assembly processes used in the industry to achieve higher production rates and lower costs. The Head Gimbal Assembly (HGA) production process has two main parts: glue dispensing and slider attaching by an Auto Core Adhesion mounting Machine (ACAM). The slider attaching process produces a mounted head to the suspension utilizing vacuum pressure to hold and position a slider. The errors from a vacuum leak from any step trigger system alarms resulting in machine downtime and slider loss defective (SLD). This paper proposes a classification algorithm derived from 250x250 micron images of mounted heads are 4 different categories: Good, Fault I, Fault II and Fault III using Convolution Neural Networks (CNN). CNN is a performance model for predictive maintenance before failure. The method has achieved a $95 \%$ accuracy for detection and classification.
\end{abstract}

Keywords-Automation machines, Fault detection and diagnosis, Machine learning, Convolutional Neural Networks.

\section{INTRODUCTION}

Automating slider attachment is a vital process leading to an increase of production output with lower costs. However, there are several process requirements in an automated control system to establish machine operation, enhance high product quality and reliability of an HDD. The HGA which performs the reading and writing of data. Fig 1-2 show the flow of ACAM process used for adhesive dispensing and slider attachment to suspension. In summary, the main process of ACAM machine is to attach the slider to the suspension per product specification. An HGA is very small. Therefore, the steps of the slider attaching process require high accuracy and precision at micron level. Fig. 2 shows the slider attachment process starting from the slider pickup which picks a slider from the sliding tray then positions it to a vacuum plate. Next step, the vacuum plate holds the slider by vacuum pressure. Then the mount head comes to pick the slider to align and attach to the suspension. The mount head uses vacuum pressure to hold the slider during transferring and mounting. As mentioned before, the vacuum pressure is a key parameter used for holding the slider. The errors from a vacuum leak at each process trigger machine alarm resulting in machine downtime and slider loss defective (SLD). The problem of a vacuum pressure case is shown in Fig. 3 illustrating surface mount head damage. Also, the vacuum leak affects the stability or even failure of ACAM machine

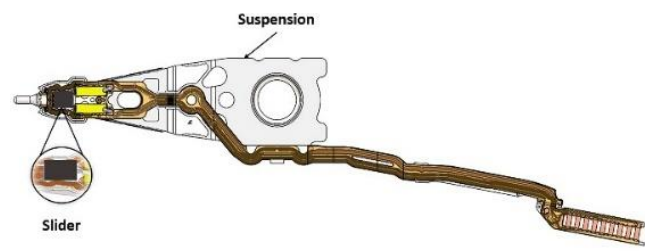

Fig. 1. Head Gimbal Assembly (HGA)

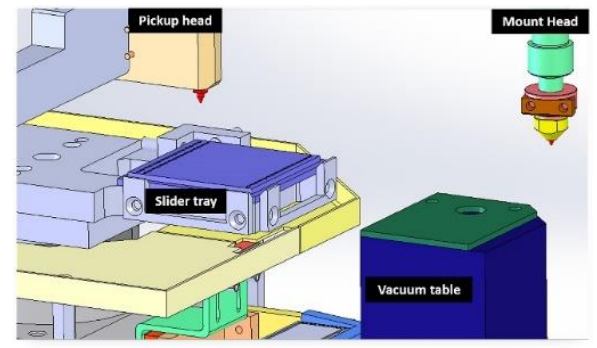

Fig. 2. Slider attachment process.

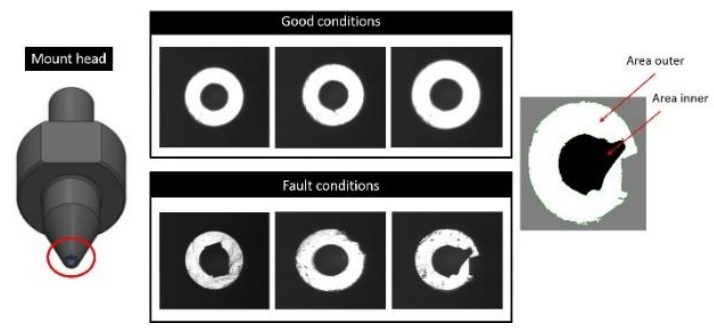

Fig. 3. Conditions surface of mount head.

An overview of this paper is as follows: Section 2 describes the literature review. Theories related to research 
are described in section 3. The proposed method of the Preprocessing images for data augmentation by Image Data Generator is described in section 4 . Section 5 describes the proposed data set and CNN architecture for presenting a model. The result and discussion are explained in section 6 . Finally, conclusion in section 7.

\section{LITERATURE REVIEW}

The review of Fault detection and diagnosis (FDD) is an essential part of modern industry to ensure safety and product quality provided by [2]. The main aim of FDI method is to raise an alarm if there is any change and to check the size, type, location and time of the occurrence of a fault in the system were proposed by [3]. In order to efficiently diagnose a leak detection for slider attaching process, Artificial Intelligence (AI) a vacuum decay for detection method is proposed to detect operating conditions when leakages occur under different conditions [5]. The Convolutional Neural Networks (CNN) ability of a strong network is excellent at representation of deep structures. The CNN is similar to Artificial Neural Networks (ANN) have a multi-layer perceptron. It consists of an input layer, hidden layer and output layer which are the inclusion of the convolutional part. This directly increases the performance of artificial neural networks. CNN has commonly been used in image classification. To investigate the vacuum leak for slider attachment process in Head Gimbal Assembly (HGA) proposed by [6]. The pixel quality/resolution of an image is vital to accurate image classification. The CNN models are built from the feature map to evaluate its performance on image recognition and detection datasets. It is an important factor in computer vision processing [7]. The techniques, i.e., feature extraction and classification method, were applied to analyze fault conditions. It was found that part of the feature learning by network that performed and classified the features using a supervised learning was developed by Dey et al. [8]

This paper proposes fault detection and diagnosis for the classification of fault levels of vacuum pressure considering the pixel image of the mount head in different conditions using Convolution Neural Network technique.

\section{RELATED WORK}

A. Fault Detection and Diagnosis

The Fault Detection and Diagnosis (FDD) consists of four key and important factors: fault isolation, fault evaluation, fault identification and fault detection. These techniques provide early warning to the system and prevent failures from occurring in the system during operation of equipment including determining the fault size, type, location and behavior at time of occurrence.

\section{B. Artificial Neural Network (ANN)}

Figure 4 shows a typical the ANN algorithm. ANN is a science of Artificial Intelligence (AI) having a similar structure and function of processing as the human brain which consists of a processor called neurons. The number of linked neurons is vastly connected and consists of 3 components as follows: input, hidden layer (can be more than 1 layer) and output. ANN is designed for regression and pattern recognition task. There are two types of learning: feed forward and back propagation as shown in Fig. 4. The ANN is suitable for regression and classification as presented by Manescu et al. [9].

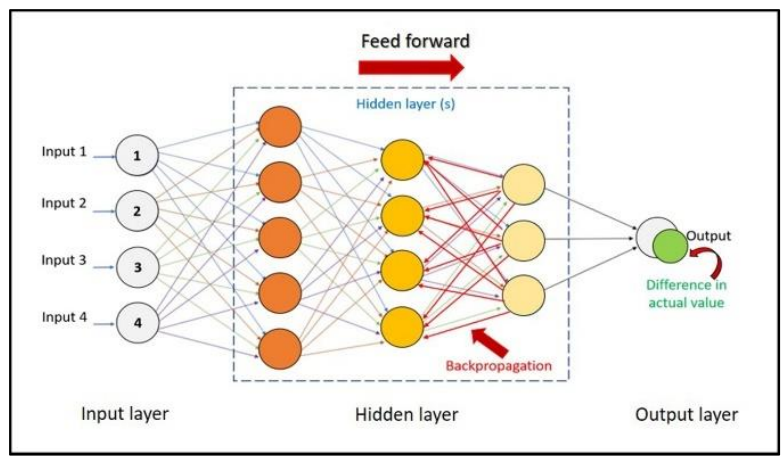

Fig. 4. Artificial Neural Network (ANN) algorithm.

Fig. 5 illustrates the activation function for process learning. The first process is to accept input of which a neuron has calculated the weight and then to enter it into transfer function. Then information is sent to activation function, a mathematical formula for making decisions and then to the next neuron or output. Most activation functions are used as logistic sigmoid function curves, also common term in statistics where $\mathrm{s}$ is the input. $\mathrm{F}$ is the output range which is limited to a range from 0 to 1 as in: Eq. 1 .

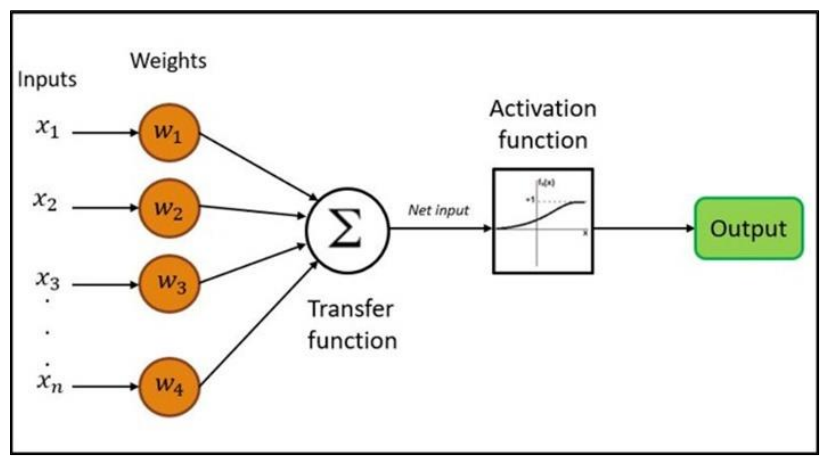

Fig. 5. The activation function for process learning

$$
\square(\square)=\frac{1}{1+\square^{-\square}}
$$

\section{CONVOLUTIONAL NEURAL NETWORK (CNN)}

The CNN is similar to the Artificial Neural Network (ANN). The network utilizes a larger model technique to determine complexity of the main objective. Thus, the main objective can be decreased the number of parameters within ANN algorithm. The input with a dimension of images passing 
through a convolution layer with activation function and pooling layer, the image becomes a feature map with shaping the feature. Pooling layer is provided to reduce parameter dimensions of the image by combining the outputs of neuron clusters at the single layer. Common pooling approach computes a max pooling or an average. Every neuron connected to another layer is called "Fully connect layer" the same as multi-layer perceptron of neuron network. In order to gain classification, SoftMax activation function is applied proposed by Bastian et al. [10] as shown in Fig. 6.

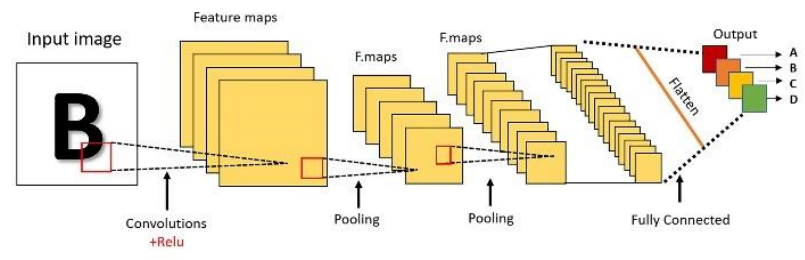

Figure 6. Artificial Convolution Neural Network (ANN) algorithm

\section{a) Convolution layer}

Convolutional is a key enabler of computer vision algorithms. The operation takes a small matrix of kernel or filler pass image input and transforms them to a feature map. The convolution feature is presented as the local image with a pixel value to determine outputs a low value. The following formula provides the output feature map show $\mathrm{G}$, input image is given $\mathrm{f}$, kernel or filter is $\mathrm{h}$ and rows/column of matrix the result are $\mathrm{m}$ and $\mathrm{n}$ as in: $\mathrm{Eq} 2$.

$$
G[m, n]=\left(f^{*} h\right)[m, n]=\sum_{j} \sum_{k} h[j, k] f[m-j, n-k]
$$

\section{b) Pooling layer}

Pooling layer is used to reduce the spatial resolution of the input image, avoid overfeeding and reduce the feature dimension by reducing computation parameters in a network. The pooling method is divided into 2 types: Average Pooling and Max Pooling. As the Average pooling layer takes the average value of the feature and the Max pooling layer chooses the maximum values from the feature map as shown in Fig. 7.

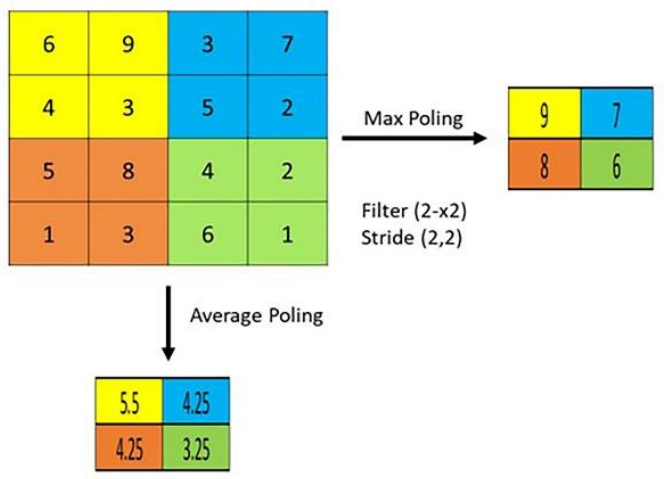

Fig. 7. Max pooling and Average pooling.
The fully connected layer is the same as a multi-layer perceptron. The neuron is connected in the network, with a single layer. This layer is applied after convolution and pooling layer to all activation functions of the previous layer is satisfied. The feature is presented with a pixel significance in the input image. In the fully connected layer, a dropout function is used to reduce the total number of parameters to avoid over-fitting that the output layer can facilitate used to classify the image.

\section{PERFORMANCE EVALUATION}

The performance model uses F1-score and accuracy for evaluating result. Accuracy is focused on the true positive and true negative of all total example. The model makes a correct prediction as in: Eq. 3. The F-1 score is a function of Precision measurement and Recall by harmonic mean as in: Eq. 4. The precision referring to positive predicted value can classify the relevant instance correctly as in: Eq. 5, the recall (result) is a good model for identifying the false negatives correctly in: Eq. 6.

$$
\begin{gathered}
\text { Accuracy }=\frac{\text { truepositives }+ \text { truenegatives }}{\text { totalexample }} \\
F 1=2 x \frac{\text { precision } x \text { recall }}{\text { precision }+ \text { recall }} \\
\text { Precision }=\frac{\text { truepositive }}{\text { truepositives }+ \text { falsepositives }} \\
\operatorname{Recall}=\frac{\text { truepositive }}{\text { truepositive }+ \text { falsenegatives }}
\end{gathered}
$$

\section{THE PROPOSED METHOD}

Fig. 8 illustrates the procedure of research for image classification. The original image has dimension of $640 \times 480$ micron. Its different aspect ratio and large parameter are not supported by CNN. Therefore, this problem can be solved by reducing image size to dimension target $250 \times 250$ micron for input to the CNN. The pixels are used in image segmentation as a group classification based on region similarity. Consider the pixels are an outer area and an inner area providing the total area of mount head images for accurate group classification. The next step is to rotate and adjust the brightness by using ImageDataGenerator method. These serve as input variable trainable to machine learning via supervised learning proposed by $\mathrm{CNN}$ algorithm that has facilitated image classification efficiently.

c) Fully Connected layer 


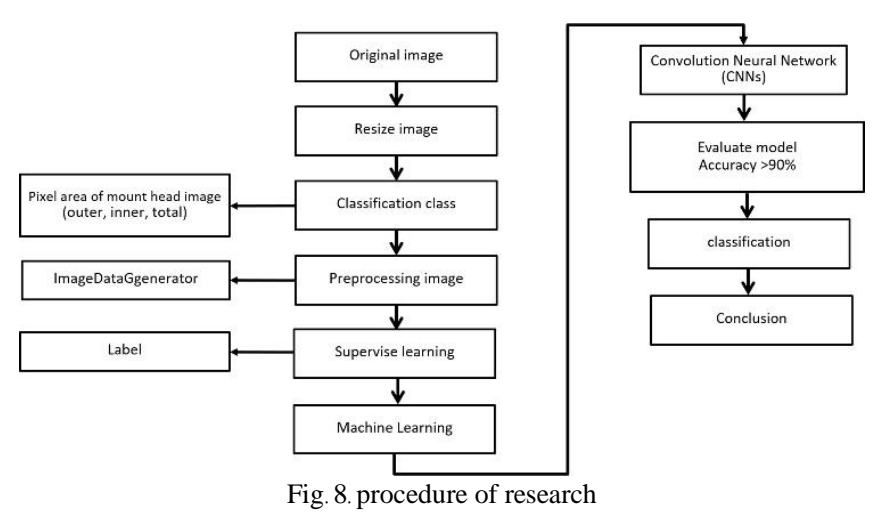

\section{A. Classification of an images}

Presented in Fig. 9 are images of a mount head showing good and fault conditions which illustrate the difference of the outer area, the inner area and the total area. The Good condition sample has a count of outer, inner and sum of mount head 18,000 - 23,000 pixels, 5,000 - 7,000 pixels and $23,000-26,000$ pixels respectively. The fault conditions consisting of Fault I, Fault II, Fault III have the pixels the outer area. The decrease in total area resulted in a fault detection. At the same time, it is found that the inner area increases in the area size, and further triggering the fault level as shown in table 1 .

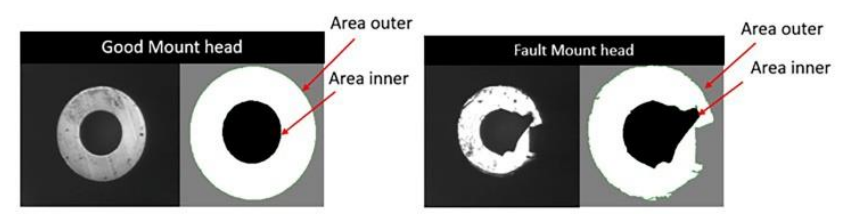

Fig. 9. Fault conditions

Table I. The pixel range for classifications conditions

\begin{tabular}{|l|c|c|c|}
\hline Conditions & $\begin{array}{c}\text { Pixel count: } \\
\text { Outer area of } \\
\text { mount head }\end{array}$ & $\begin{array}{c}\text { Pixel count: } \\
\text { Inner area of } \\
\text { mount head }\end{array}$ & $\begin{array}{c}\text { Pixel count: } \\
\text { Total area of } \\
\text { mount head }\end{array}$ \\
\hline Good & $18,000-23,000$ & $5,000-7,000$ & $23,000-26,000$ \\
\hline Fault I & $17,000-18,000$ & $5,600-9,000$ & $22,000-24,000$ \\
\hline Fault II & $15,000-17,000$ & $5,000-8,000$ & $21,000-23,000$ \\
\hline Fault III & Less than 15,000 & More than 8,000 & Less than 21,000 \\
\hline
\end{tabular}

\section{B. Processing images}

- Cropping image to reduce parameter

The original images have a $640 \times 480$ pixels dimension is a very different ratio dimensions in height $\mathrm{x}$ width and large as the pixel as shown in Fig. 10 (a). that is a multiplying factor by the CNN algorithm. Therefore, presenting a cropped and resized image reduces spatial size by covering only the areas of interest which have a target dimension of 250x250 micron. shows a resized image Fig. 10 (b).

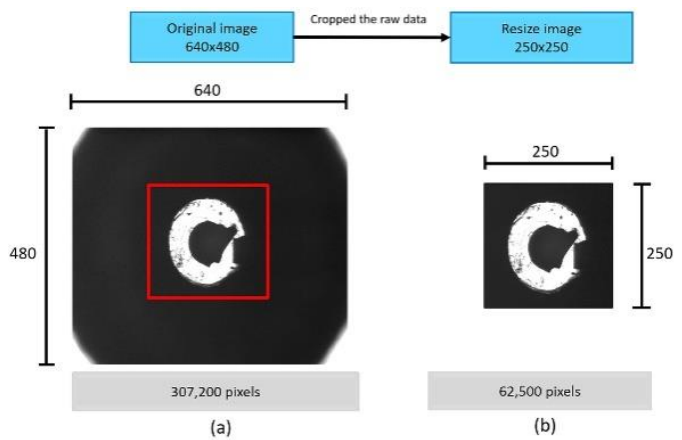

Fig. 10. Cropping to resize an image

\section{- $\quad$ Rotation image and Brightness}

Data augmentation is conducted by the artificially creating new data for featured data sets that are applied to the images after being cropped and resized. The operation of pre-processing images by rotating them $45^{\circ}, 90^{\circ}$ and $180^{\circ}$ with horizontal flip and vertical flip are shown in Fig. 11. After cropping and rotating them, we adjust the brightness with the random increase and decrease applied as shown in Fig. 12. In order to increase the size of the data set, the preprocessing method - Image DataGenerator method proposed by Keras is considered.

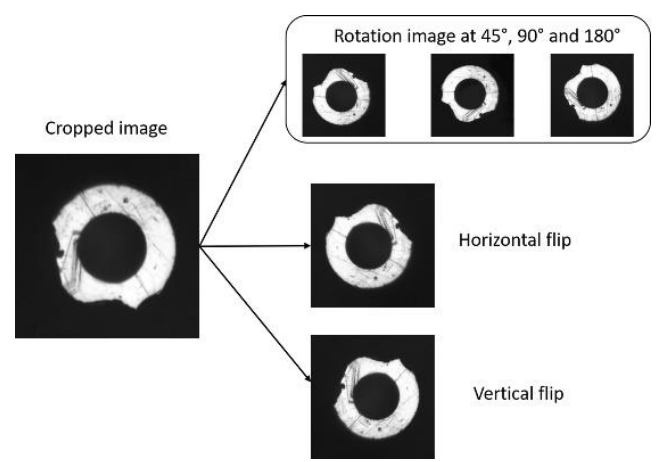

Figure 11. Transformed image from rotation.
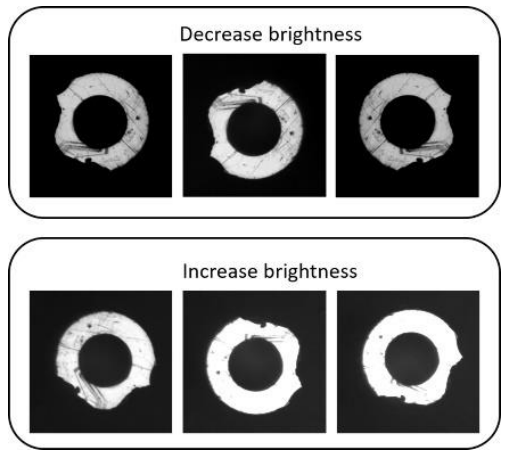

Figure 12. Transformed image from brightness

\section{- Data set}

To increase the sample size of database, transformation has been applied to the cropped, rotated and brightness adjusted images. The original dataset consists of 500 images and then pre-processed, in which 1 image contains 24 additional images from rotation, increase light and decrease light. The images dataset increases from 500 to 12,500 images for 
training, validation and test in the model. Split $80 \%$ data into training, 20\% for testing and validation of model learning via supervise learning. The training and test set distribution is planned based on 10,000 trained images and testing 1,250 images. Each 250x250 micron pixels image size is applied as input parameter to the model and has 4 output class labels (Good, Fault I, Fault II and Fault III).

\section{CLASSIFIER MODEL}

\section{A. Dataset}

Mount head is the dataset used for image recognition of fault level. The original dataset has 500 images. Fig. 13 shows the ImageDataGenerator method to increase the images dataset from 500 to 12,500 images for training and testing the model. $80 \%$ and $20 \%$ of data were split into training testing and validating respectively. The model learning via supervise learning divided 4 output class labels: Good, Fault I, Fault II and Fault III.

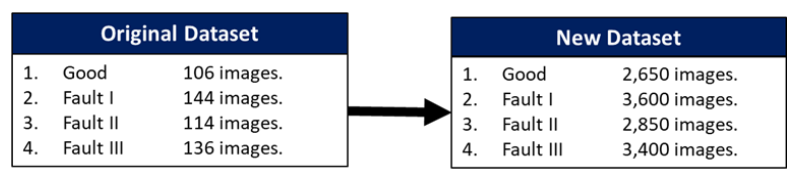

Fig. 13. Generate images of dataset.

\section{B. CNN model}

The architecture of the model input images which is resized as $250 \times 250 \times 3$ ( 250 wide, 250 high, 3 color channels), would lead to neurons that have $250 * 250 * 3=187,500$ weights. The size of the filter is set to $3 \times 3$ with same padding and max pooling is $2 \times 2$ for all convolutional layers. RELU activation function is a standard for all hidden layers. The network model is 3 layers of the convolution network, and they flatten at same multi-perceptron layer into the fully connected network with 0.2 dropout to the next layers. The last part is SoftMax layer for classifying output. The process flow model of CNN is shown in Fig. 14.

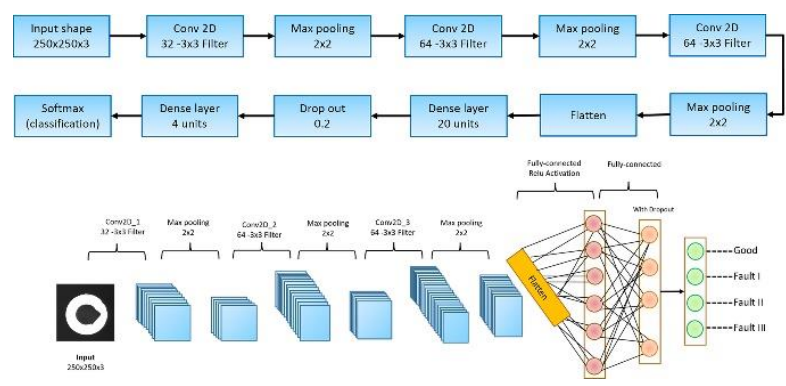

Fig. 14. Model of Convolutional Neural Network (CNN).

\section{ANALYSIS AND DISCUSSION}

The CNN model accuracy represents classification accuracy of results for the training of the 200 epochs (number of training data). Following convolutional method consists of kernel filter, max-pooling and fully connected layers. The model is applied on mount head dataset of which dimension is $250 \times 250$ micron sized with RGB images of 4 classes, 10,000 training images and 1,250 testing images. Figure 16 shows an achieved accuracy of $97.05 \%$ training and $98.56 \%$ validation for 4 classes of the mount head images dataset. Fig. 16 shows the result of model loss which was a correlation between accuracy for training and test method resulting in loss of 0.0722 and 0.0364 respectively. The ability to classify the image of the mount head is clearly demonstrated. A result ensures that the fault classification level is significantly effective. By defining the conditions of images with Fault I [ 1,0,0,0], Fault II [0,1,0,0], Fault III $[0,0,1,0]$ and Good [0,0,0,1]. Fig. 17 (a) further demonstrates the accurate prediction condition of an image from a mount head. The prediction of false images will mostly happen with Good and II conditions predicted as Fault I. Since there are faults between the two groups, there are images in the input that are quite similar to the grouping in the pixel classification as shown in Fig. 17 (b).

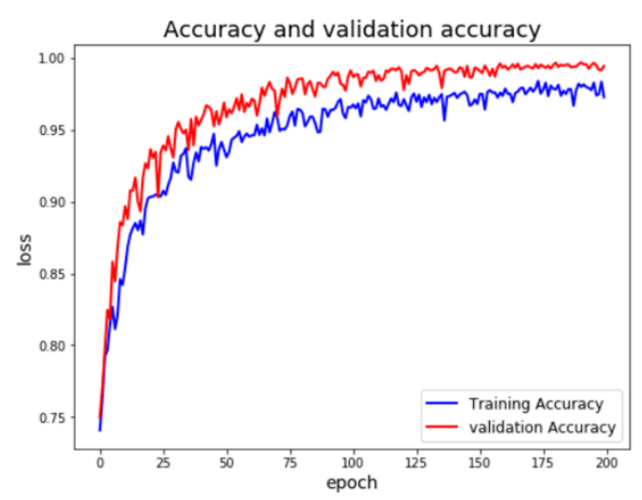

Figure 16. Result of model accuracy and model loss.

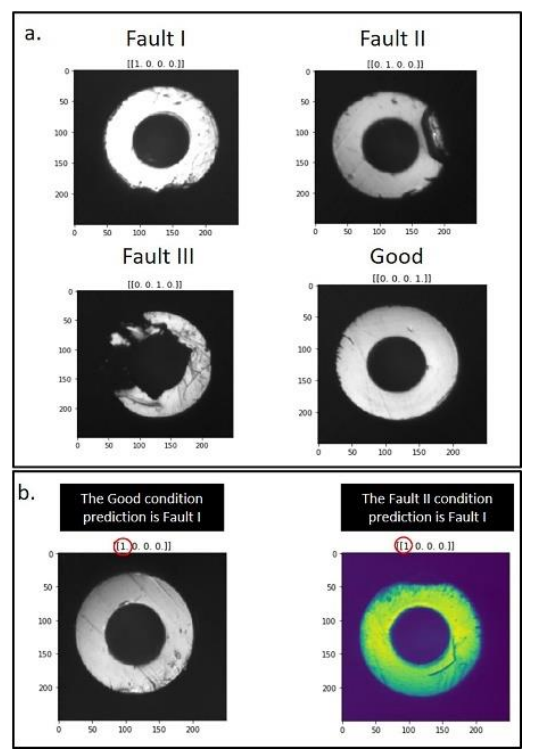

Fig. 17. The predicted condition of mount head.

\section{CONCLUSION}

In this work, the Fault Detection and Diagnosis using CNN architectures for efficiency in the classification of mount 
head images was proposed. The CNN achieved an accuracy of $97.05 \%$ training and $98.56 \%$ validation for 4 classes of the mount head image dataset. The classification accuracy was near $100 \%$. It classified the dataset into four classes: Good, Fault I, Fault II, and Fault III

\section{ACKNOWLEDGMENT}

This research was supported by the Western Digital (Thailand) Co., Ltd. Thank you for providing a scholarship to study a Master's degree at Suranaree University of Technology (SUT).

\section{References}

[1] Chommaungpuck, P., Lawbootsa, S. and Srisertpol, J., 2019. Fault detection of linear bearing in auto core adhesion mounting machine using artificial neural network. WSEAS Transactions on Systems and Control, 14, pp.31-42

[2] Deeying, J., K. Asawarungsaengkul, and P. Chutima. "Multi-objective optimization on laser solder jet bonding process in head gimbal assembly using the response surface methodology." Optics \& Laser Technology 98 (2018): 158-168.
[3] Chauhan, Vedang, and Brian Surgenor. "A comparative study of machine vision based methods for fault detection in an automated assembly machine." Procedia Manufacturing 1 (2015): 416-428

[4] Thirumarimurugan, M., N. Bagyalakshmi, and P. Paarkavi. "Comparison of fault detection and isolation methods: A review." 2016 10th International Conference on Intelligent Systems and Control (ISCO). IEEE, 2016.

[5] Krysander, Mattias, and Erik Frisk. "Leakage detection in a fuel evaporative system." Control engineering practice 17.11 (2009): 12731279.

[6] Veeriah, Vivek, Naifan Zhuang, and Guo-Jun Qi. "Differential recurrent neural networks for action recognition." Proceedings of the IEEE international conference on computer vision. 2015.

[7] Chauhan, Rahul, Kamal Kumar Ghanshala, and R. C. Joshi. "Convolutional Neural Network (CNN) for Image Detection and Recognition." 2018 First International Conference on Secure Cyber Computing and Communication (ICSCCC). IEEE, 2018.

[8] Dey, D., et al. "A deep learning framework using convolution neural network for classification of impulse fault patterns in transformers with increased accuracy." IEEE Transactions on Dielectrics and Electrical Insulation 24.6 (2017): 3894-3897.

[9] Manescu, Petru, et al. "Accurate and interpretable classification of microspectroscopy pixels using artificial neural networks." Medical image analysis 37 (02017): $37-45$

[10] Bastian, Blossom Treesa, et al. "Visual inspection and characterization of external corrosion in pipelines using deep neural network." NDT \& E International 107 (2019): 102134

\section{Creative Commons Attribution License 4.0 (Attribution 4.0 International, CC BY 4.0)}

This article is published under the terms of the Creative Commons Attribution License 4.0 https://creativecommons.org/licenses/by/4.0/deed.en_US 